\title{
Exact Geometric Relationships, Symmetry Breaking and Structural Stability for Single- Walled Carbon Nanotubes
}

\author{
Tong Zhang*, Ze Shuai Yuan, Li Hao Tan
}

(Received 29 August; accepted 28 November; published online 2 December.)

\begin{abstract}
We pioneered a study about how the geometric relationship of single-walled carbon nanotubes (SWCNT) is influenced by curvature factor and non-planar geometry factor in cylindrical coordinate system based on the assumption of complete symmetry. The bond length and angle of every carbon-carbon bonds are determined by using the principle of the minimum energy. The results of the paper include: (1) From the calculation result, the symmetry breaking appears for chiral carbon nanotubes, while the part symmetry appears for achiral carbon nanotubes with increasing curvature. (2) The synergistic effect of bond lengths and bond angles is first found. (3) We conclude that the influence of non-planar geometry factor can be completely ignored on bond lengths and bond angles when the curvature parameter has been included in the model. (4) The two fractal dimensions are given from the nanoscale to the macroscale for zigzag topology and armchair topology respectively. Fractal dimensions of SWCNT show special characteristics, varying with the length of SWCNT until the lengths approach infinity. The close and inevitable correlations among curvature, symmetry breaking and stability of SWCNTs can be summed up as: the increase of curvature causes symmetry breaking, and such symmetry breaking will further reduce the structural stability.
\end{abstract}

Keywords: Geometry; Structural Stability; Symmetry Breaking; Fractal Dimension

Citation: Tong Zhang, Ze Shuai Yuan and Li Hao Tan, "Exact Geometric Relationships, Symmetry Breaking and Structural Stability for Single-Walled Carbon Nanotubes", Nano-Micro Lett. 3 (4), 228-235 (2011). http://dx.doi.org/10.3786/nml.v3i4.p228-235

\section{Introduction}

The relationships among geometric parameters, physical structure and structural stability for singlewalled carbon nanotubes (SWCNT) have not been explored in details. When building mechanics and other related models, characterizing the geometric parameters of SWCNT accurately has become the origin and foundation of investigating the symmetry breaking and structural stability. In fact, the geometric relationships have been discussed in literature [1-5], in which the geometric structures of SWCNT are usually assumed to be planar. The curvature influence on the geometric relationship is generally ignored in such kind of models
[1-5], thereby resulting in errors.

Based on the spatial geometry and structure of SWCNT, Cox and Hill [1] deduced the threedimensional geometric relationships rather accurately from the exact geometric computation, with the analytical results providing a valuable reference for error analysis. Although the curvature has been considered in the process of establishing the geometric model, bond lengths and bond angles of carbon nanotubes are assumed to be equal in advance. It can be expected that such geometric parameters would not match well with the real structure and physical characterization of SWCNT. For example, the error of an opposite angle at the center is at least in the order of $n^{-5}$. Fur- 
thermore, the error would increase greatly when $\mathrm{n}$ decreases. With the potential energy between atoms characterized by Brenner potential energy, geometrical parameters could be calculated based on the principle of minimum energy by Jiang et al. [6]; and then the geometric relationships of SWCNT are deduced very accurately by considering the curvature influence.

The geometric relationships are actually described based on the planar assumption, neglecting the fact that the points A, B, C, D are not in the same plane but on a curved surface for the real structure, as shown in Fig. 1(c). Therefore the vectors which are not in the same plane originally are also treated as those in the same plane. Meanwhile, the perimeter of the section of SWCNT, which is composed of a series of secant lines but was treated as an arc in the model [6], is represented by a chiral parameter $C_{h}$. Therefore, when the spatial position of each carbon atom is calculated, the following relationship is obtained:

$$
Z_{A}=a_{4} \sin \left(\varphi_{2}+\theta\right), \quad \Theta_{A}=\frac{2 a_{4} \cos \left(\varphi_{2}+\theta\right)}{d_{t}}
$$

Where $\varphi_{2}+\theta$ would also result in the corresponding error because both of them are not in a same plane.

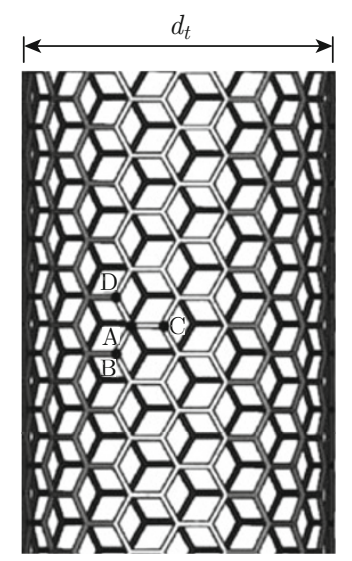

(a) Three-dimensional model of carbon nanotubes

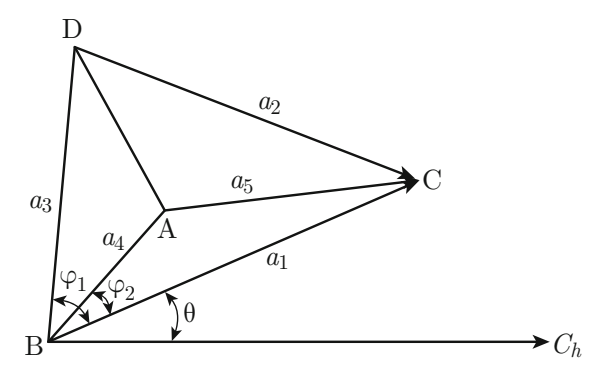

(c) Free

Fig. 1 Planar and three-dimensional model of SWCNT.

\section{New Model for the Real Structure}

\section{Geometric description}

A SWCNT can be regarded as a single graphene sheet rolled up in such way to form a hollow cylinder, and all of carbon atoms are embedded on the cylindrical surface, as shown in Fig. 2. We find that that determining the spatial position of an atom in carbon nanotubes under the cylindrical coordinate system is more convenient than traditional characterization methods using the Cartesian coordinate system. Moreover, any carbon atom is regarded the same as any other atom due to the assumption of complete symmetry.

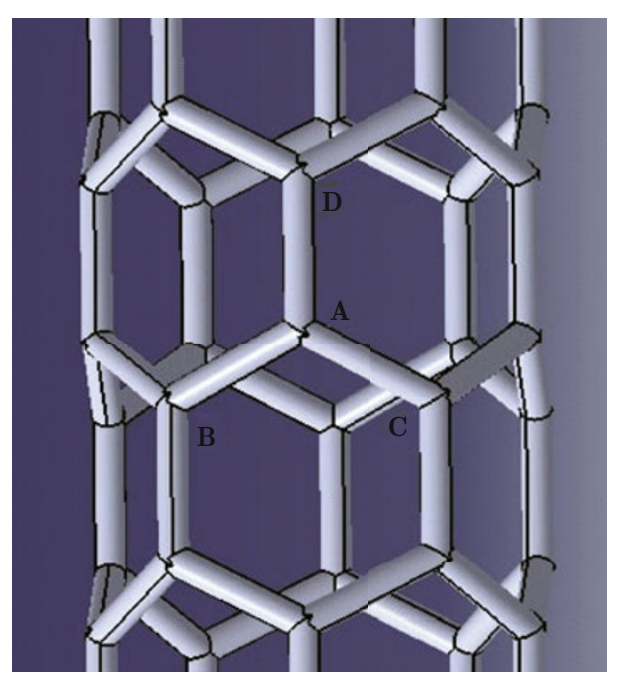

Fig. 2 A novel model for SWCNT under the cylindrical coordinate system, point A corresponding to the central atom, and point $\mathrm{B}, \mathrm{C}, \mathrm{D}$ corresponding to the neighboring atoms.

An assumption is made for the new model that each carbon atom in the cylindrical section has the same weight when SWCNT is kept stable. In order to express the geometric relationship of SWCNT more accurately, $\mathrm{C}-\mathrm{C}$ bonds are treated as secant lines which connect each point, instead of the arcs. Each point corresponds to a carbon atom on the cylindrical surface as shown in Fig. 2.

The central axis of the cylinder coincides with axial direction of a SWCNT in the cylindrical coordinates. The spatial position of each carbon atom can be represented by two independent parameters $(\Psi, Z)$. Radius of carbon nanotubes - $r_{0}$ is defined as the distance between the atom and the central axis, thereby the position of each atom could be expressed into $\left(r_{0} \cos \Psi, r_{0} \sin \Psi, Z\right)$.

Without loss of generality, assuming that carbon atom A lies on the origin of the coordinate system, 
where $\Psi_{A}=0, Z_{A}=0$, then:

$$
\begin{aligned}
& \overrightarrow{A B}=\left(r_{0}\left(\cos \Psi_{B}-1\right), r_{0} \sin \Psi_{B}, Z_{B}\right) \\
& \overrightarrow{A C}=\left(r_{0}\left(\cos \Psi_{C}-1\right), r_{0} \sin \Psi_{C}, Z_{C}\right) \\
& \overrightarrow{A D}=\left(r_{0}\left(\cos \Psi_{D}-1\right), r_{0} \sin \Psi_{D}, Z_{D}\right)
\end{aligned}
$$

And then other two vectors can be obtained, as shown in Fig. 2:

$$
\overrightarrow{B C}=\overrightarrow{A C}-\overrightarrow{A B}, \quad \overrightarrow{D C}=\overrightarrow{A C}-\overrightarrow{A D}
$$

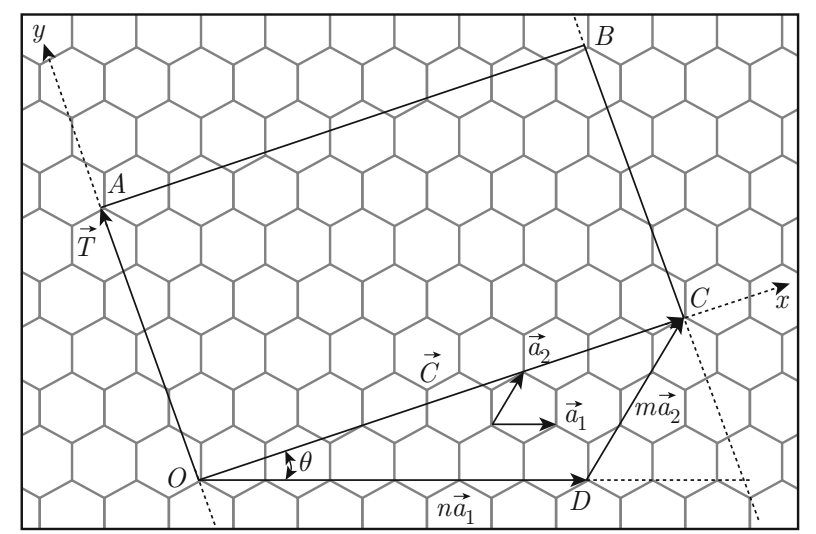

Fig. 3 Diagram of the chiral vector $(\mathrm{n}, \mathrm{m})$ for SWCNT, rolled up from a graphene.

According to chiral parameters for carbon nanotubes, a SWCNT is divided and expanded along the generatrices. Lines $\mathrm{OA}$ and $\mathrm{CB}$ are the generatrices on the

cylindrical surface, as shown in Fig. 3. From vector $\overrightarrow{\vec{C}}=n \vec{a}_{1}+m \vec{a}_{2}$, it will reach the spatial position of the original atom A. Vectors $\vec{a}_{1}$ and $\vec{a}_{2}$ correspond to vectors $O D$ and DC respectively, as shown in Fig. 3.

Under the cylindrical coordinate system, the distance from point $\mathrm{B}$ to point $\mathrm{C}$ corresponds to the increment $\left(\Psi_{C}-\Psi_{B}, Z_{C}-Z_{B}\right)$. Similarly, the distance from point $\mathrm{D}$ to point $\mathrm{C}$ corresponds to the increment $\left(\Psi_{C}-\Psi_{D}, Z_{C}-Z_{D}\right)$.

Therefore the following geometric relationship can be established:

$n\left(\Psi_{C}-\Psi_{B}, Z_{C}-Z_{B}\right)+m\left(\Psi_{C}-\Psi_{D}, Z_{C}-Z_{D}\right)=(2 \pi, 0)$

By expanding the upper formula:

$$
\begin{aligned}
& \Psi_{B}=\left(\frac{m}{n}+1\right) \Psi_{C}-\frac{m}{n} \Psi_{D}-\frac{2 \pi}{n} \\
& Z_{B}=\left(\frac{m}{n}+1\right) Z_{C}-\frac{m}{n} Z_{D}
\end{aligned}
$$

Thus, the free parameters, by which spatial positions of carbon atoms in SWCNT could be described, are reduced to five parameters: $\Psi_{C}, \Psi_{D}, Z_{C}, Z_{D}, r_{0}$.

The $\mathrm{C}-\mathrm{C}$ covalent bond lengths and angles can be expressed by these five parameters:

$$
r_{X Y}=\sqrt{ } 2 r_{0}^{2}\left[1-\cos \left(\Psi_{X}-\Psi_{Y}\right)\right]+\left(Z_{X}-Z_{Y}\right)^{2}
$$

$$
\begin{aligned}
\theta_{X Y Z} & =\arccos \frac{\overrightarrow{r_{X Y}} \cdot \overrightarrow{r_{X Z}}}{r_{X Y} r_{X Z}} \\
& =\arccos \quad r_{0}^{2}\left[\cos \left(\Psi_{Y}-\Psi_{Z}\right)-\cos \Psi_{Y}-\cos \Psi_{Z}+1\right]+Z_{Y} Z_{Z} \\
& \sqrt{ } 2 r_{0}^{2}\left[1-\cos \left(\Psi_{X}-\Psi_{Y}\right)\right]+\left(Z_{X}-Z_{Y}\right)^{2} \sqrt{ } 2 r_{0}^{2}\left[1-\cos \left(\Psi_{X}-\Psi_{Z}\right)\right]+\left(Z_{X}-Z_{Z}\right)^{2}
\end{aligned}
$$

where suffix $X, Y, Z$ represent the random three of four carbon atoms A, B, C, D, as shown in Fig. 4.

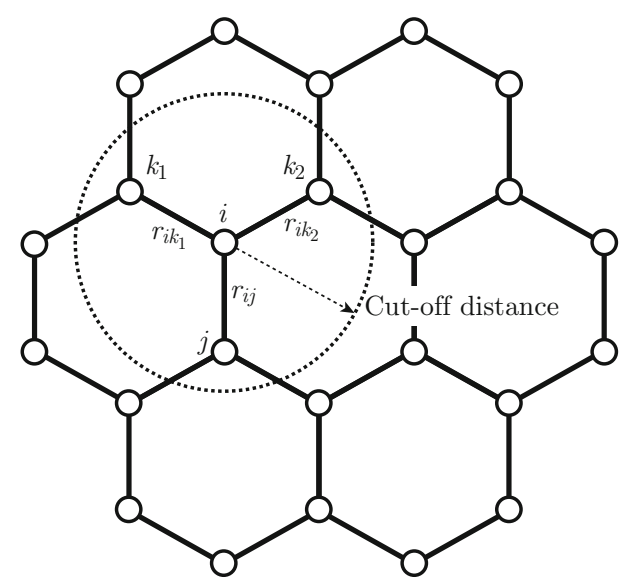

Fig. 4 Schematic of atomic structure of graphene

\section{Interatomic potential energy equation}

Based on the effects of multi-body atomic interactions in SWCNT, Richard et al. [3] have given the expression for potential energy:

$$
V\left(r_{i j}\right)=V_{R}\left(r_{i j}\right)-B_{i j} V_{A}\left(r_{i j}\right)
$$

where $i, j$ represent a pair of carbon atoms which form a covalent bond, and $r_{i j}$ is the covalent bond length; $V_{R}$ and $V_{A}$ determined only by bond lengths are expressed in the formula below:

$$
\begin{aligned}
& V_{R}(r)=\frac{D^{(e)}}{S-1} e^{-\sqrt{2 S} \beta\left(r-R^{(e)}\right)} f_{c}(r) \\
& V_{A}(r)=\frac{D^{(e)} S}{S-1} e^{-\sqrt{\frac{2}{S}} \beta\left(r-R^{(e)}\right)} f_{c}(r)
\end{aligned}
$$

where $D^{(e)}, S, \beta, R^{(e)}$ are the characteristic parameters. The function $f_{c}(r)$ is used to restrict the influence range 
between carbon atoms by the choice of $R^{(1)}$ and $R^{(2)}$ :

$$
f_{c}(r)= \begin{cases}1 & r<R^{(1)} \\ \frac{1}{2}\left\{1+\cos \left[\frac{\pi\left(r-R^{(1)}\right)}{R^{(2)}-R^{(1)}}\right]\right\} & R^{(1)}<r<R^{(2)} \\ 0 & r>R^{(2)}\end{cases}
$$

$B_{i j}$ in equation (8) represents the covalent bond energies influenced by the other carbon atoms outside the bond. It depends on the environment where carbon atoms $\mathrm{i}$ and $\mathrm{j}$ are located:

$$
B_{i j}=\left[1+\sum_{k \neq i, j} G\left(\theta_{i j k}\right) f_{c}\left(r_{i k}\right)\right]^{-\delta}
$$

where $k$ represents other carbon atoms except $i$ and $j$, and $\theta_{i j k}$ is the angle between covalent bonds from the same atom $i$ to two other atoms $k$ and $j$, as shown in Fig. 5. $G$ is expressed as follows:

$$
G(\theta)=a_{0}\left[1+\frac{c_{0}^{2}}{d_{0}^{2}}-\frac{c_{0}^{2}}{d_{0}^{2}+(1+\cos \theta)^{2}}\right]
$$

where $\delta, a_{0}, c_{0}, d_{0}$ are constants.

For the atomic structure of SWCNT, the above constants are given as below:

$D^{(e)}=6.000 \mathrm{eV}, \quad S=1.22, \quad \beta=21 \mathrm{~nm}^{-1}$,

$R^{(e)}=0.1390 \mathrm{~nm}, \quad R^{(1)}=0.17 \mathrm{~nm}, \quad R^{(2)}=0.20 \mathrm{~nm}$,

$\delta=0.50000, \quad a_{0}=0.00020813, \quad c_{0}=330, \quad d_{0}=3.5$

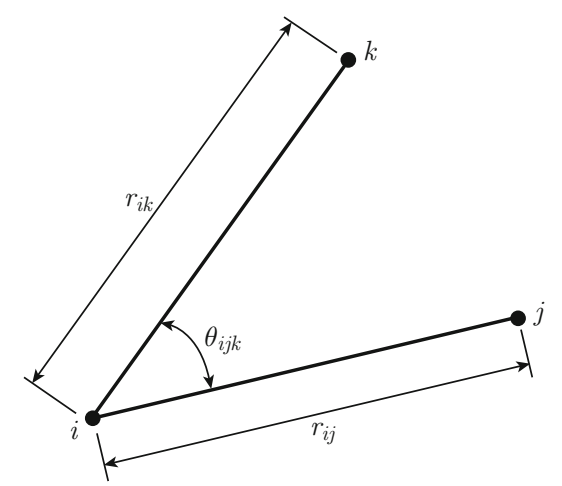

Fig. 5 Carbon atoms $i, j$ and $k$, the corresponding bonds $i-j$ and $i-k$, and bond angle

\section{Geometric relationships for the most stable structure}

Five parameters $\Psi_{0}, \Psi_{D}, Z_{C}, Z_{D}, r_{0}$ are used to express the relevant bond lengths and angles, and then the whole potential energy can be calculated by substituting them into expression (8). Around the central atom A, covalent energy $V\left(r_{A B}\right), V\left(r_{A C}\right), V\left(r_{A D}\right)$ can be obtained, and then the structure of a SWCNT would meet minimum energy requirements. The following formula can be acquired for meeting the stability of a SWCNT:

$$
V=V\left(r_{A B}\right)+V\left(r_{A C}\right)+V\left(r_{A D}\right)=\min
$$

where $V=V\left(\Psi_{C}, \Psi_{D}, Z_{C}, Z_{D}, r_{0}\right)$. The partial derivatives of $\mathrm{V}$ with respect to the five free variables can be calculated, and make all of them equal to zero, the below equation can be given:

$$
\frac{\partial V}{\partial \Psi_{C}}=\frac{\partial V}{\partial \Psi_{D}}=\frac{\partial V}{\partial Z_{C}}=\frac{\partial V}{\partial Z_{D}}=\frac{\partial V}{\partial r_{0}}=0
$$

The values of the five independent variables in the extreme points would be solved. The equation itself is highly nonlinear and transcendental, so its solving procedure is too complicated to obtain the analytic solution. Based on the simplex method to solve minimum value of the multivariate function using MATLAB toolbox [7], the value of the free variables $\left(\Psi_{C}, \Psi_{D}, Z_{C}, Z_{D}, r_{0}\right)$ can be calculated directly on the condition that $V=\min$, then all the $\mathrm{C}-\mathrm{C}$ bond lengths and angles could be obtained. Lastly, the five free variables will be substituted into $V\left(\Psi_{C}, \Psi_{D}, Z_{C}, Z_{D}, r_{0}\right)$ to calculate the whole potential energy, which would meet the principle of least potential energy spontaneously if a SWCNT could keep the stable structure.

\section{Computational results and discussion}

\section{Symmetry breaking of chiral SWCNT and part symmetry of achiral SWCNT}

It can be concluded that bond lengths $\mathrm{AB}$ and $\mathrm{AC}$ overlap completely, as shown in Fig. 6 (a.1), and bond angles ABD and ACD overlap completely, as shown in Fig. 6 (a.2), once chiral parameter is determined for the zigzag-type SWCNT. In a similar way, bond lengths $\mathrm{AB}$ and $\mathrm{AD}$ overlap completely as shown in Fig. 6 (b.1) and bond angles $\mathrm{ABC}$ and $\mathrm{ACD}$ overlap completely as shown in Fig. 6 (a.2) once chiral parameter is determined for the armchair-type SWCNT. However, all three bond lengths and bond angles are no longer the same, showing geometric breaking symmetry, as shown in Fig. 6 (c.1) and Fig. 6 (c.2) for the chiral SWCNT.

Fig. 6 Comparison of all three bond lengths and angles for zigzag-type, armchair-type and chiral SWCNT respectively

There appears part symmetry of bond lengths and bond angles for the achiral SWCNT, but no symmetry at all for the chiral SWCNT when each element of the chiral parameter is lower than parameter $(25,9)$ respectively. This means that two equal bond lengths and bond angles appear for achiral SWCNT, which include zigzag-type and armchair-type SWCNT, however, geometric symmetry breaking appears for chiral SWCNT. 


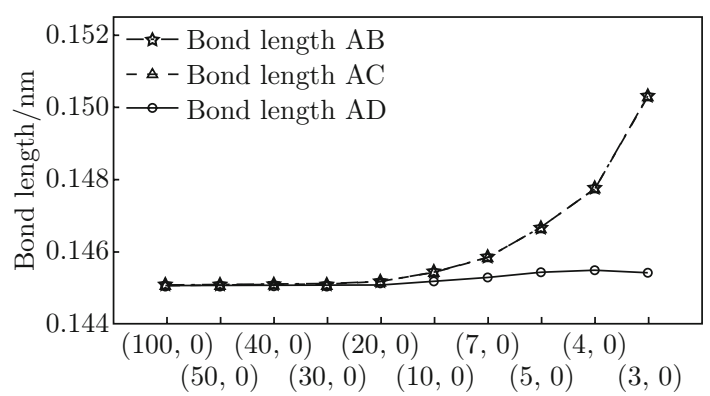

Chiral paramenters

(a.1) Zigzag nanotubes bond lengths

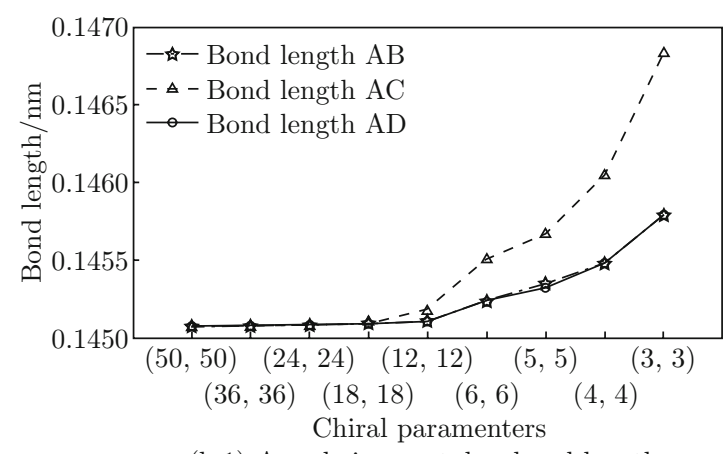

(b.1) Armchair nanotubes bond lengths

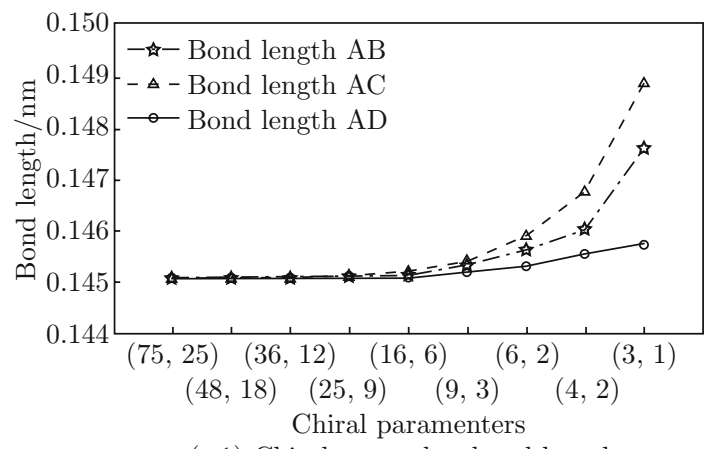

(c.1) Chiral nanotubes bond lengths

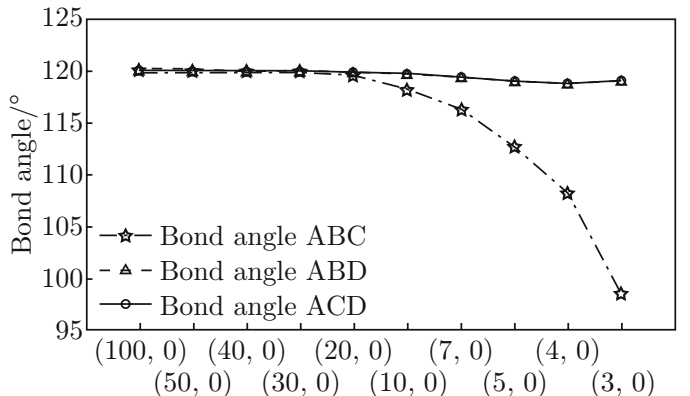

$(50,0) \quad(30,0) \quad(10,0) \quad(5,0)$
$\quad$ Chiral paramenters

(a.2) Zigzag nanotubes bond angles

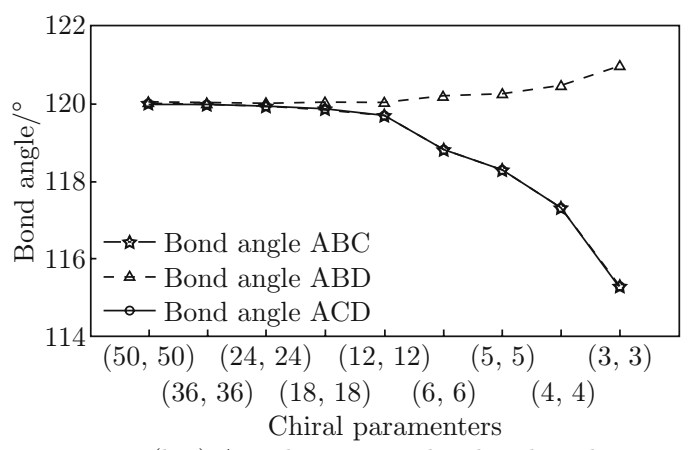

(b.2) Armchair nanotubes bond angles

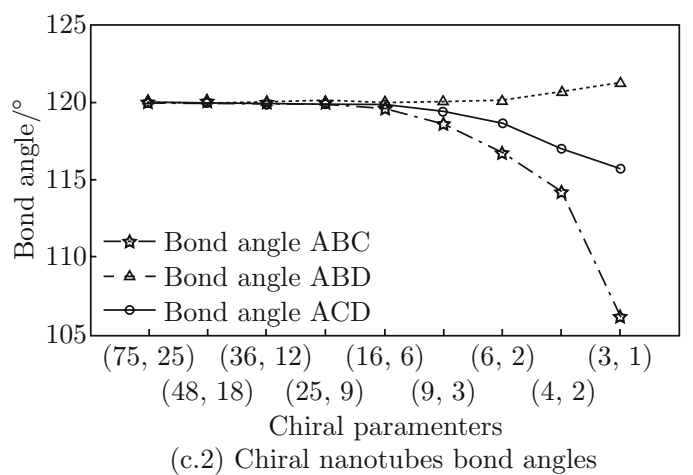

Fig. 6 (a.1) Illustration of differences among three bond lengths for zigzag-type SWCNT; (a.2) Illustration of differences among three bond angles for zigzag-type SWCNT; (b.1) Illustration of differences among three bond lengths for armchairtype SWCNT; (b.2) Illustration of differences among three bond angles for armchair -type SWCNT; (c.1) Illustration of differences among three bond lengths for chiral SWCNT; (c.2) Illustration of differences among three bond angles for chiral SWCNT.

This is the essential difference of the geometric characteristic between chiral and achiral SWCNT. Previous literature shows the geometric characteristic based on geometric intuition and assumption, however, this study proved by using the numerical method for the first time.

The symmetrical bond lengths and bond angles always keep symmetry respectively. That is to say, the symmetry remains changeless. In addition, the variance in bond angles synchronizes with that in bond lengths. All three bond angles lie in the asymmetric spatial position at the same time when all three bond lengths lie in the asymmetric spatial position. Otherwise, when all three bond lengths are exactly equal, all three bond angles are also exactly equal. From a geometrical point of view, these phenomena show the synergistic effect between bond lengths and bond angles in SWCNT for the first time.

\section{The differences of both bond lengths and bond angles induced by the curvature}

The differences in bond lengths and bond angles are computed for SWCNT of different chirality, as shown in Fig. 6. The abscissa from left to right corresponds to the change of chiral parameters from big to small. In addition, as the number of carbon atoms decreases along circumferential direction of SWCNT, the surface curvature increases, so the abscissa from left to right corresponds to the curvature change from small 
to large.

With decreasing chirality parameter, differences in bond lengths and bond angles show a gradual increase. The reason is that the curvature influence on bond lengths and bond angles would not be ignored any more for high curvature magnitude. Consequently, the conclusion could be drawn that all three equal bond lengths and bond angles in SWCNT is wrong for high curvature magnitude or decreasing chirality parameter. This conclusion coincides with the results [1].

\section{Error influence by non-planar geometry factor}

Further calculations show that with a certain chiral critical point, or a certain critical curvature, the differences in bond lengths and bond angles are over $0.01 \%$ and $0.1 \%$ respectivly, as shown in Table 1 . From these data, influence of non-planar geometry on the bond angles is greater than that on the bond lengths for high curvature magnitude.

Table 1 Comparison of the relative errors in bond lengths and bond angles with different chiral parameters

$\begin{array}{cccc} & \begin{array}{c}\text { Chiral } \\ \text { parameters }\end{array} & \begin{array}{c}\text { Relative } \\ \text { error in } \\ \text { bond lengths }\end{array} & \begin{array}{c}\text { Relative } \\ \text { error in } \\ \text { bond angles }\end{array} \\ \begin{array}{c}\text { zigzag } \\ \text { nanotubes }\end{array} & (20,0) & 0.006 \% & 0.304 \% \\ \text { armchair } & (24,24) & 0.027 \% & 1.244 \% \\ \text { nanotubes } & (18,18) & 0.009 \% & 0.065 \% \\ \text { chiral } & (25,9) & 0.012 \% & 0.119 \% \\ \text { nanotubes } & (16,6) & 0.039 \% & 0.070 \% \\ & & & 0.186 \%\end{array}$

Meanwhile, there are three corresponding critical chiral parameters for $(20,0)$ zigzag SWCNT, $(24,24)$ armchair SWCNT and $(25,9)$ chiral SWCNT respectively. When each element of chiral parameter is over the corresponding critical chiral parameters respectively, the relative errors in all three bond lengths and bond angles would be less than $0.01 \%$ and $0.1 \%$ respectively. Obviously, non-planar geometry factor may be ignored in such cases. Under these conditions, the traditional assumption that all three bond lengths and bond angles are equal would be true [1]. The reason lies in that curvature is small enough for the four points A, B, C, $\mathrm{D}$ assumed to be located in the same plane, thereby all three bond lengths and bond angles are equal.

The calculated bond lengths and bond angles in this study are compared with those reported by Jiang et al. [6], as shown in Fig. 7. The relative errors in bond lengths and bond angles of chiral SWCNT are taken as an example. There is very little difference in bond lengths and bond angles with and without considering the non-planar geometry factor. The results agree well with the prediction [1].
From Fig. 7, we observe that the relative errors between the two models are below $0.025 \%$. It has been proven that non-planar geometry influence on bond lengths and bond angles could be neglected if the curvature parameter had been considered in advance.
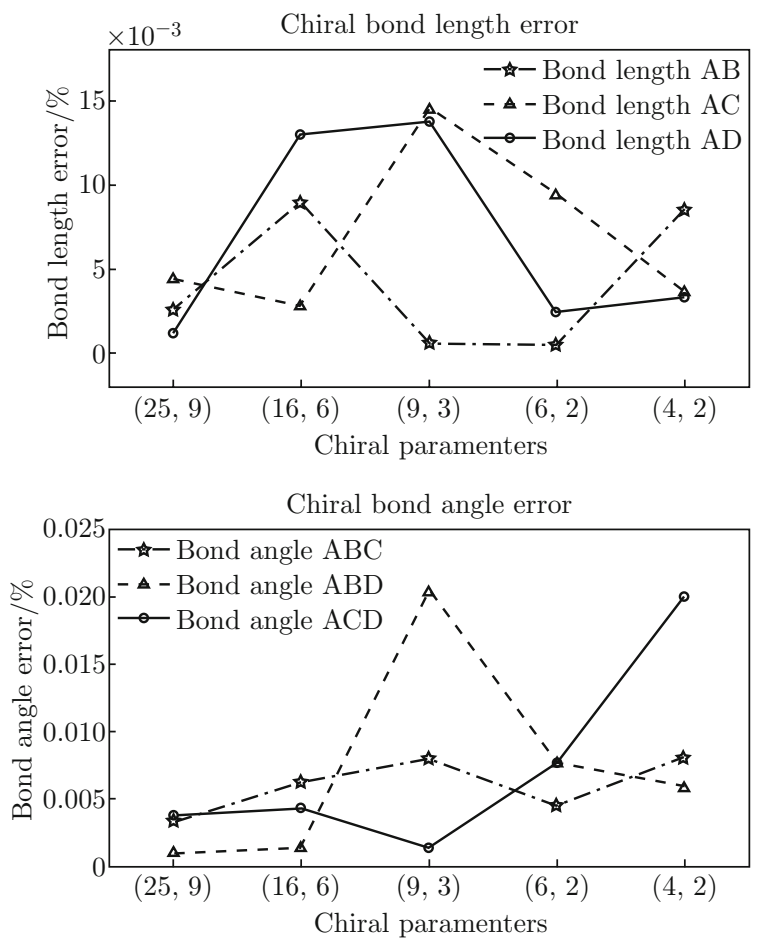

Fig. 7 Relative errors are compared with results [6] in bond lengths and bond angles by considering the non-planar geometry factor respectively.

\section{The structural stability}

For different SWCNT, structural stability could be measured by the average potential energy of a $\mathrm{C}$ atom. The lower the average potential energy, the higher is the stability. The structural stabilities of three different types of SWCNT are compared in Fig. 8, where the reciprocal of nanotube radius of the SWCNT is used to represent the curvature value.

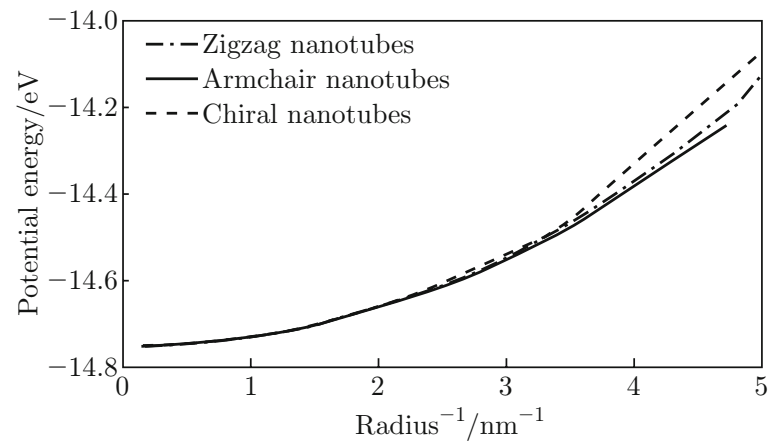

Fig. 8 Relation curve between curvature value and the potential energy of a $\mathrm{C}$ atom with three different types of SWCNT. 
The conclusions are:

(1) The divergences of bond lengths and bond angles increase with the increasing curvature respectively. The absolute symmetry of achiral SWCNT is broken with the increasing curvature for non-planar geometry factor and then evolves into the part symmetry. Compared with achiral SWCNT, there is no symmetry at all and more weakening trend towards symmetry for the chiral SWCNT with the increasing curvature.

(2) The geometric symmetry is closely related to the structural stability. The higher the geometric symmetry, the higher is the structural stability. Consequently, geometric symmetry breaking appears with the increasing curvature, and furthermore leads to the increase in the potential energy of a $\mathrm{C}$ atom, which causes the stability of the structure to become lower. That is to say, achiral SWCNT still have the trend of maintaining the part symmetry of the structure spontaneously-even when the potential energy is beyond the minimum potential. As for chiral SWCNT, once the potential energy is beyond the minimum potential, the whole symmetry of the structure would be lost entirely.

(3) The potential energy of a $\mathrm{C}$ atom is related to the curvature directly. It can be said that the curvature is the primary factor to break the geometrical symmetry. When the curvature indefinitely approaches 0 , the potential energy of a $\mathrm{C}$ atom approaches $-14.75 \mathrm{eV}$, which is the minimum potential energy of a $\mathrm{C}$ atom in graphene. It can be concluded that: (a) the minimum potential energy of a $\mathrm{C}$ atom in SWCNT is constant regardless of different types of SWCNT with lower curvature between 0.3 and 2.2 , as shown in Fig. 8 . On the other hand, the differences in structural stability emerge when the curvature value surpasses 3.4. (b) chiral SWCNT is the most unstable structure, followed by zigzag type SWCNT, and armchair type SWCNT is most stable structure when the curvature surpasses 3.4, as shown in Fig. 8.

In summary, there is a close and inevitable correlation among curvature, asymmetry and stability for SWCNTs.

\section{The fractal dimensions of achiral SWCNT}

Due to its relative convenience in mathematical calculations and empirical estimations, box counting method is one of the most widely used methods in calculating the fractal dimension of a fractal set. However, this method is difficult to apply for many fractal sets in three-dimensional space [8]. Therefore, the application of this method in SWCNT has not been reported yet. A SWCNT with self-similarities in geometry make the box counting method feasible and efficient to determine the fractal dimension by a special covering set a common tube to cover SWCNT, instead of traditional covering set such as a ball or cubes in $R^{3}$ [9].
A SWCNT is a regular fractal set in Riemann space, butits fractal dimension shows special characteristics. With the aid of box counting method [9], fractal dimensions of the armchair type and zigzag type SWCNT in all scales are provided in Fig. 10 and Fig. 11 respectively. The two fractal dimensions vary with the length of the SWCNT respectively until the lengths of SWCNT approach infinity. At the nano scale, the fractal dimensions lie between 1.8 and 2.3 and at the macro scale, the fractal dimensions approach 1 like onedimensional material.

As for the armchair carbon nanotubes, if the number of the Y-branched junctions along the axial direction is fixed, then the larger the number of the Y-branched junctions along the circumferential direction and the higher the fractal dimension becomes, having a smaller curvature value, as shown in Fig. 9. Similarly, this applies to the zigzag carbon nanotubes, as shown in Fig. 10.

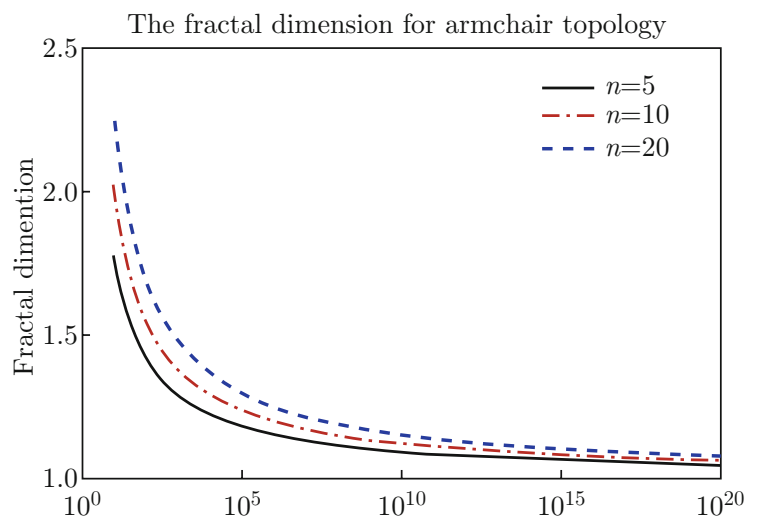

The numbers of Y-branched junctions along the axial direction

Fig. 9 The relationship curve between fractal dimension for armchair topology from the numbers of the Y-branched junctions along the axial direction and the numbers of the Y-branched junctions along the circumferential direction

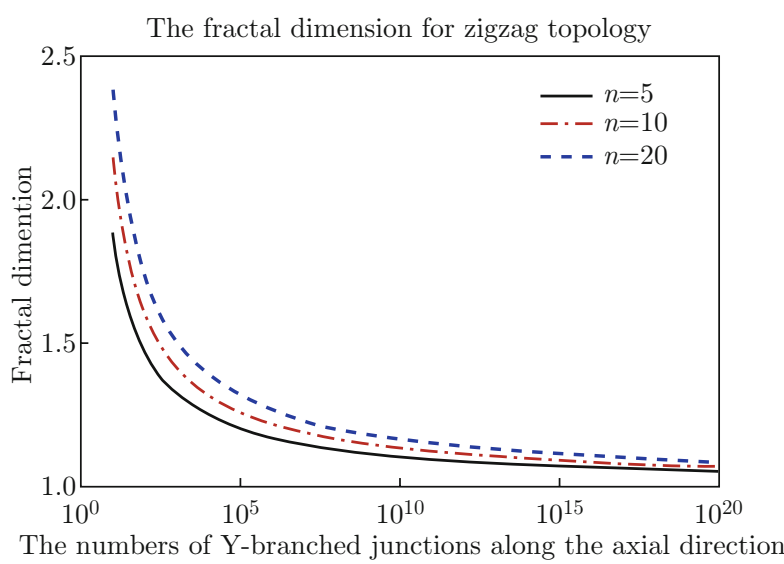

Fig. 10 The relationship curve between fractal dimension for zigzag topology from the numbers of the Y-branched junctions along the axial direction and the numbers of the Y-branched junctions along the circumferential direction 


\section{Conclusions}

Geometry is complex in carbon nanotubes. The electronic and mechanical properties of a carbon nanotube are intrinsically linked with its geometry, in particular, its curvature and symmetry. Describing the geometry of carbon nanotubes is the first step towards carrying out the relevant fundamental research and other engineering applications. For example, some errors may be avoided in the calculation of mechanical properties of SWCNT by the introduction of the exact geometrical conditions. In another example, an atomistic description of hydrogen adsorption mechanisms in SWNT requires a clear understanding of tube geometry. In addition, the determination of hydrogen storage feasibility and the optimization of geometrical variables are codependent goals. The symmetry breaking and structural stability of SWCNT, combined with geometry, are another problem of fundamental importance, to which it has not been fully paid attention by scientists today. Investigation of the relationship among geometric relationships, symmetry breaking and structural stability of SWCNT would lay a basic foundation for engineering applications of SWCNT.

The main conclusions in this study may include:

(1) The existence of geometrical symmetry has been proven for SWCNT by performing the numerical experiments for the first time. It breaks away traditional geometric intuition and assumption in previous research.

(2) For the real structure of SWCNT, all three bond lengths and bond angles are not equal, and the respective difference among all three lengths and bonds would not be neglected with decreasing chiral parameters (n, $\mathrm{m})$ or with increasing curvature.

(3) The model in this paper is established by considering non-planar geometry factor. It has been proven that non-planar influence on calculated bond lengths and bond angles could be ignored from numerical experiments if the curvature parameter had been considered in advance.

(4) Part symmetry appears for the zigzag and armchair carbon nanotubes, but no symmetry appears at all for chiral SWCNT with increasing curvature. This is the another essential difference of the geometric characteristic between chiral and achiral structure of SWCNT from a geometric point of view. Furthermore, the synergistic effect of bond lengths and bond angles is found. These geometrical rules have been proven and elucidated for the first time in this study.

(5) There is close and inevitable correlation among curvature, symmetry breaking and structural stability for SWCNT. Structural stability is constant regardless of different types of SWCNT with lower curvature between 0.3 and 2.2. There is very little difference between structural stability of different types of SWCNT with middle curvature between 2.2 and 3.4. While chiral topology is the most unstable structure, followed by zigzag topology, the armchair topology is the most stable structure when the curvature value surpasses 3.4.

(6) From the fractal geometry point of view, its fractal dimension for carbon nanotubes shows special characteristics. The two fractal dimensions for zigzag and armchair vary with the length of nanotube respectively until the lengths approach infinity. At the nano scale, the fractal dimensions lie between 1.8 and 2.3. At the macro scale, both of the two fractal dimensions approach 1. The full range of results achieves a complete unified understanding on the fractal dimensions of SWCNT, bridging the nano to macro scale gap.

\section{Acknowledgments}

The authors thank National Natural Science Foundation of China (No. 10602028) and Student Research Train Program of BeiHang University.

\section{References}

[1] B. J. Cox and J. M. Hill, Carbon 45, 1453 (2007). http://dx.doi.org/10.1016/j. carbon. 2007.03.028

[2] R. Setton, Carbon 33, 135 (1995). http://dx.doi. org/10.1016/0008-6223(94)00117-I

[3] K. F. Richard, B. J. Cox and J. M. Hill J. Math. Chem. 47, 569 (2010). http://dx.doi.org/10.1007/ s10910-009-9586-5

[4] D. Baowan, B. J. Cox and J. M. Hill, J. Math. Chem. 44, 515 (2008). http://dx.doi.org/10.1007/ s10910-007-9325-8

[5] V. K. Jindal and A. N. Imtani, Comp. Mat. Sci. 44, 156 (2008). http://dx.doi.org/10.1016/j. commatsci. 2008.01.020

[6] H. Jiang, P. Zhang, B. Liu, Y. Huang, P. h. Geubelle and H. Gao, et al, Comp. Mat. Sci. 28, 429 (2003). http://dx.doi.org/10.1016/j. commatsci.2003.08.004

[7] Z. Y. Zhang and Z. Y. Yang, "MATLAB Tutorial", Press of Beijing University of Aeronautics and Astronautics (2002).

[8] Kenneth Falconer, 2rd ed. Chichester, John Wiley \& Sons Ltd (2003).

[9] T. Zhang, G. W. Tang, Y. J. Yin, X. Feng and Z. Lu, J. Nanosci. Nanotech. 7, 1 (2010). http://dx.doi.org/ 10.1504/IJNT . 2010. 029546 